\title{
Association Between Gut Akkermansia and Metabolic Syndrome is Dose-Dependent and Affected by Microbial Interactions: A Cross-Sectional Study
}

\author{
Qi Zhou (D) ${ }^{1, *}$ \\ Guofang Pang ${ }^{2, *}$ \\ Zhirong Zhang ${ }^{3}$ \\ Huiping Yuan' \\ Chen Chen' \\ Nan Zhang' \\ Ze Yang' \\ Liang Sun ${ }^{1,4}$
}

'The Key Laboratory of Geriatrics, Beijing Institute of Geriatrics, Beijing Hospital, National Center of Gerontology, National Health Commission, Institute of Geriatric Medicine, Chinese Academy of Medical Sciences, Beijing, People's Republic of China; ${ }^{2}$ Guangxi Jiangbin Hospital, Nanning, Guangxi, People's Republic of China; ${ }^{3}$ Fengtai Center for Disease Control and Prevention, Beijing, People's Republic of China; ${ }^{4} \mathrm{NHC}$ Key Laboratory of Drug Addiction Medicine, Kunming Medical University, Kunming, People's Republic of China

*These authors contributed equally to this work

\begin{abstract}
Objective: Akkermansia muciniphila is among the most abundant bacterial species in the human intestine; however, its relationship to metabolic syndrome (MetS) - which is linked to gut dysbiosis-is not known. In this study, we investigated the association between Akkermansia abundance and risk of MetS and its components, as well as dose-response effects and the influence of microbial interactions on the association.
\end{abstract}

Methods: This cross-sectional study included 6896 Chinese participants aged 18 to 97 years from the Guangdong Gut Microbiome Project. MetS was defined according to Joint Committee for Developing Chinese Guidelines on Prevention and Treatment of Dyslipidemia in Adults criteria. The abundance of Akkermansia was assessed by $16 \mathrm{~S}$ rRNA sequencing. Logistic regression analysis with adjustment for common confounders was performed to evaluate the association between Akkermansia and MetS and its components. Models with restricted cubic splines and interaction terms were used to examine the dose-response association and microbial interactions, respectively.

Results: The prevalence of MetS was 20.4\%, and the median abundance of Akkermansia was $0.08 \%$ (interquartile range: $0.04-0.93 \%$ ). Increased Akkermansia abundance was associated with decreased risk of MetS ( $\left.P_{\text {nonlinear }}<0.05\right)$, but this effect was not observed until the Akkermansia level was $0.2 \%$ of the total gut microbiota abundance (odds ratio $=0.96,95 \%$ confidence interval: 0.94-0.98). Of the $5 \mathrm{MetS}$ components, obesity and hypertriglyceridemia showed the strongest association with Akkermansia, followed by reduced high-density lipoprotein cholesterol, hypertension, and hyperglycemia. Microbial interaction analyses showed that Ruminococcaceae and Lachnospiraceae were the predominant bacterial families and were not only correlated with Akkermansia abundance but also influenced the Akkermansia-MetS association.

Conclusion: There is a dose-response association between reduced risk of MetS and increased abundance of Akkermansia. The association between Akkermansia and 5 MetS components is variable and affected by microbial interactions.

Keywords: metabolic syndrome, gut microbiota, Akkermansia muciniphila, dose-response, microbial interaction

\section{Introduction}

Metabolic syndrome (MetS) — which is characterized by central obesity, insulin resistance, hypertension, and dyslipidemia — is a major global health problem. ${ }^{1}$ Evidence from epidemiologic and physiologic studies indicates that metabolic disorders are closely linked to gut dysbiosis. ${ }^{2,3}$ Specifically, changes in microbial diversity and composition can alter intestinal permeability, ${ }^{4}$ induce low-grade inflammation, ${ }^{5}$ and influence liver gluconeogenesis by stimulating the production of short-chain fatty 
acids. ${ }^{6}$ Gut microbes are potential targets in the treatment of metabolic diseases. The role of some probiotics including Faecalibacterium prausnitzii, Akkermansia muciniphila, and Clostridium spp. in metabolic disorders has been explored in animal models and humans. ${ }^{7}$

A. muciniphila is among the most abundant bacterial species in the human intestine and is a potentially beneficial microbe in the treatment of metabolic disease. ${ }^{8-10}$ Although the protective effects of $A$. muciniphila against MetS components such as obesity and hyperglycemia have been investigated, ${ }^{7,11}$ there have been no studies on the association between Akkermansia and the 5 main MetS components. One study reported that administration of A. muciniphila for 3 months had variable effects on MetS components, with decreases in fat mass and hip circumference but no changes in fasting glycemia and triglyceride levels compared to a placebo. $^{12}$

Gastrointestinal homeostasis depends on the gut microbiome. ${ }^{7}$ A probiotic can only exert beneficial effects if it can colonize its host under competition and cooperation with native microbiota. ${ }^{13}$ Akkermansia is a mucindegrading bacterium that resides in the mucus layer of the gut; ${ }^{14}$ its effect on host physiology depends on complex interactions with other intestinal microorganisms. Indeed, depletion of Akkermansia along with enrichment of other mucolytic bacteria such as Fusobacterium spp. has been reported in both humans and animal models of inflammatory bowel disease. ${ }^{11}$ However, it remains unclear which microorganisms affect the association between Akkermansia and MetS; moreover, the threshold level of Akkermansia required to reduce the risk of MetS is not known. Clarifying these points has important clinical implications for the treatment of MetS through modulation of Akkermansia and the gut microbiome.

In this cross-sectional study of 6896 subjects from the Guangdong Gut Microbiome Project (GGMP), ${ }^{15,16}$ we analyzed the association between Akkermansia in the gut and 5 components of MetS, and examined the doseresponse effect and influence of the gut microbiome on this association.

\section{Materials and Methods Data Sources}

This was a cross-sectional study based on data from the GGMP, ${ }^{15,16}$ which aimed to establish the generalizability of microbiota-based diagnostic models for metabolic disease. Participant data were obtained from stool and blood samples and a questionnaire. The GGMP was approved by the Ethical Review Committee of the Chinese Centre for Disease Control and Prevention (approval no. 201519-A) with written, informed consent obtained from each participant.

For stool samples, participants were given a stool sampler, ice bag, and ice box to collect and transport their samples within 1 day to a nearby collection point where there were $-20^{\circ} \mathrm{C}$ freezers for sample storage. The Fecal DNA Bead Isolation kit (Bioeasy, Shenzhen, China) was used to extract total bacterial DNA; the 16S rRNA gene V4 region was amplified with barcoded primers (V4F, 5'GTGYCAGCMGCCGCGGTAA-3' and V4R, 5'GGACTACNVGGGTWTCTAAT2-3') and used to construct a sequencing library. Paired-end sequencing was performed on the HiSeq2500 system (HiSeq SBS Kit v4; Illumina, San Diego, CA, USA).

Raw sequencing reads (https://github.com/ SMUJYYXB/GGMP) and metadata (https://github.com/ SMUJYYXB/GGMP) were downloaded from the European Nucleotide Archive (accession no. PRJEB18535). Metadata consisted of MetS-related risk factors including systolic blood pressure (SBP), diastolic blood pressure (DBP), fasting plasma glucose (FPG), triglyceride (TG), high-density lipoprotein cholesterol (HDL-c), and waist circumference; potential confounders were age, sex, Bristol stool scale, geographic location, annual income, antibiotic consumption, and diet (plant or animal oil, fruits, grains, synbiotics, and vegetables).

\section{Definitions of MetS and Its Components}

MetS and its risk components were defined according to the criteria of the Joint Committee for Developing Chinese Guidelines on Prevention and Treatment of Dyslipidemia in Adults ${ }^{17}$ as follows: 1 ) hypertension, SBP $\geq 130 \mathrm{mmHg}$ and/ or DBP $\geq 85 \mathrm{mmHg}$ and/or use of antihypertensive drugs; 2 ) hyperglycemia, FPG $\geq 6.1 \mathrm{mmol} / 1$ and/or use of blood glucose-lowering medication and/or previous diagnosis of type 2 diabetes mellitus (T2DM); 3) hypertriglyceridemia, TG $\geq 1.70 \mathrm{mmol} / 1$ and/or use of triglyceride-lowering medication; 4) reduced high-density lipoprotein cholesterol (HDLc) $(<1.04 \mathrm{mmol} / \mathrm{l})$; and 5) central obesity, waist circumference $\geq 90 \mathrm{~cm}$ in men and $\geq 85 \mathrm{~cm}$ in women. MetS was diagnosed when a subject had at least 3 of the 5 metabolic risk components.

\section{Assessment of Akkermansia Abundance}

Raw 16S rRNA gene sequencing reads were split according to an online script (pairend.extract_sequences.pl, 
https://github.com/SMUJYYXB/GGMP) provided by the GGMP project. Paired-end reads were merged with FLASH 1.2.11 ${ }^{18}$ and qualified (with a maximum error rate of 0.01) with VSEARCH 2.1.4.2. ${ }^{19}$ Filtered reads were clustered into operational taxonomic units (OTUs) with a similarity of $97 \%$. Representative OTUs were classified into taxonomic groups (sintax_cutoff, 0.8) based on the Silva_16s_v123 dataset. $^{20}$ OTUs present in $<10 \%$ of samples were excluded to reduce the computational load, leaving $98.54 \%$ (834 OTUs) of the initial abundance. Analyses were performed at the OTU or genus level.

\section{Statistical Analysis}

Descriptive statistics and comparisons are reported as mean \pm standard deviation (SD) or median with interquartile range (IQR) for continuous variables, or as percentages for categorical variables. Trend tests for variables across different quintiles of Akkermansia abundance were based on linear regression analysis or the CochranArmitage test. The Mann-Whitney $U$-test and chisquared test were used to evaluate intergroup differences for continuous and categorical variables, respectively.

Logistic or multivariate linear regression was used to examine the associations between Akkermansia and MetS and its components. Models were first adjusted for age and sex and then further adjusted for Bristol stool scale, geographic location, and annual income. Trend tests of odds ratio (OR) and 95\% confidential intervals (95\% CI) across quintiles were conducted by entering the median value of each quintile of Akkermansia abundance into the models.

Cubic splines with 4 knots at the 5th, 25th, 75th, and 95th percentiles were used to evaluate the dose-response relationship between Akkermansia and the risk of MetS and its components. Nonlinearity of the associations was identified by testing whether the coefficients associated with the nonlinear components were equal to zero. ${ }^{21}$ Fitted possibilities of outcomes for different levels of Akkermansia were used to visualize the shape of doseresponse curves. In parallel, we separated Akkermansia abundance into 20 quantiles, estimated the ORs (compared to the first percentile [Q1]) from the lowest to the highest quantile, and analyzed the change in MetS risk with increasing Akkermansia abundance as a line plot. Finally, we assessed the reproducibility of the models by changing parameters such as the position of knots for cubic splines and potential confounders for the later model.

We investigated the effects of interactions between Akkermansia and other microbes on MetS in 3 steps. 1)
We identified Akkermansia-correlated and MetSassociated microbes by multivariate linear regression with adjustment for age and sex. MetS components were treated as continuous response variables and the abundance of OTUs was arcsine-square root-transformed. A significant association was defined when the coefficient of OTUs in the model had a false discovery rate (FDR) $<0.05$. 2) We estimated the multiplicative interactions between Akkermansia and other OTUs (obtained from step 1 by logistic regression). OTUs with a meaningful interaction coefficient term $(P<0.05)$ were considered to significantly influence the Akkermansia-MetS association and were identified as interacting (i)OTUs. 3) The iOTUs in step 2 were grouped according to change in OR ( $\triangle \mathrm{OR})$. Microbial associations with Akkermansia OTUs were plotted with Circos 0.69-9. The "interactions" package of $\mathrm{R}$ software was used to assess the change in the Akkermansia-MetS association when iOTU abundance increased with SD or from the Q1 to Q5.

All data analyses were performed using $\mathrm{R} \times 64 \mathrm{v} 4.0 .0$. Statistical significance was evaluated with 2-tailed tests using a cutoff value of $P \leq 0.05$. For multiple testing, FDR adjustment was performed with the BenjaminHochberg method.

\section{Results}

\section{Characteristics of Study Participants}

This study included 6896 participants aged 18-97 years. The average age was $52.7 \pm 14.6$ years and $55.2 \%$ of participants were female. The median relative abundance of Akkermansia was 0.08\% (IQR: 0.04-0.34\%) and 97.7\% of participants had at least $0.01 \%$ Akkermansia. The prevalence of MetS was $20.4 \%$ and that of its components were as follows: central obesity, 23.8\%; hyperglycemia, 18.1\%; hypertension, 50.6\%; hypertriglyceridemia, $23.7 \%$; and reduced HDL-c, $28.8 \%$.

Table 1 shows the demographic and clinical characteristics of the study participants across quintiles of Akkermansia. Young females had a higher abundance of Akkermansia, and participants with a higher abundance had a lower prevalence of MetS, central obesity, hyperglycemia, hypertension, hypertriglyceridemia, and reduced HDL-c. Likewise, Akkermansia level was inversely related to DBP, SBP, waist circumference, HDL-c, and TG $(\mathrm{FDR}<0.05)$ in both crude and sex- and age- adjusted models (Supplementary Table 1 and Supplementary 
Table I Characteristics of Participants According to Quintile of Akkermansia Abundance $(n=6896)$

\begin{tabular}{|c|c|c|c|c|c|c|}
\hline Characteristics & QI & Q2 & Q3 & Q4 & Q5 & $P$ for Trend \\
\hline $\mathrm{n}$ & 1379 & 1379 & 1379 & 1379 & 1380 & \\
\hline Akkermansia $^{\mathrm{a}}$, (\%) & $0.02(0.02-0.03)$ & $0.04(0.04-0.05)$ & $0.08(0.06-0.09)$ & $0.20(0.15-0.33)$ & $7.05(2.04-18.93)$ & \\
\hline Age, years, mean(sd) & $53.6(14.5)$ & $53.7(14.6)$ & $52.0(14.5)$ & $51.4(14.6)$ & $52.9(15.0)$ & $<0.001$ \\
\hline Sex, male, n (\%) & $621(45.0)$ & $623(45.2)$ & $653(47.4)$ & 661 (47.9) & $535(38.7)$ & 0.02 \\
\hline MetS, n (\%) & $263(19.1)$ & $288(20.9)$ & $326(23.6)$ & $303(22.0)$ & $224(16.23)^{* *}$ & 0.18 \\
\hline Central obesity, n (\%) & $34 \mid(24.7)$ & $330(23.9)$ & $370(26.8)$ & $341(24.7)$ & $259(18.8)^{* *}$ & $<0.001$ \\
\hline Hyperglycemia, n (\%) & $225(16.3)$ & $262(19.0)$ & $274(19.9)$ & $252(18.3)$ & $234(16.9)^{* *}$ & 0.86 \\
\hline Hypertension, n (\%) & $7 \mid 2(5 \mid .6)$ & $739(53.5)$ & $703(51.0)$ & $694(50.3)$ & $638(46.2)$ & $<0.001$ \\
\hline Hypertriglyceridemia, n (\%) & $300(21.8)$ & $328(23.8)$ & $366(26.5)$ & $396(28.7)$ & $243(17.3)^{* *}$ & 0.35 \\
\hline Low HDL, n (\%) & $376(27.3)$ & $401(29.1)$ & $417(30.2)$ & $434(31.5)$ & $356(25.8)^{* *}$ & 0.88 \\
\hline
\end{tabular}

Notes: ${ }^{a}$ Median (interquartile range) relative abundances of Akkermansia. **Data of Q5 was significantly different from those of QI, Q2, Q3 and Q4 $(P<0.00 \mathrm{I})$.

Figure 1). However, the association between Akkermansia and FBG was nonsignificant.

We also investigated the associations between Akkermansia and potential confounders (Supplementary Figure 2). The abundance of Akkermansia differed across geographic regions $(\mathrm{FDR}=0.001)$. However, stool type; a diet that included plant or animal oil, fruits, grains, synbiotics and vegetables; annual income; and consumption of antibiotics were not significantly correlated with Akkermansia.

\section{Associations Between Akkermansia and MetS and Its Components}

The relative abundance of Akkermansia was inversely associated with the risk of MetS; a $1 \%$ increase in Akkermansia reduced the risk of MetS by $\sim 0.3 \%$ (Figure $1 \mathrm{~A}$ and Supplementary Table 2). Compared to Q1, ORs (95\% CIs) for MetS from the lowest to the highest quintiles of Akkermansia abundance were as follows: Q2, 1.04 (0.89-1.20); Q3, 1.27 (1.11-1.47); Q4, 1.13 (0.98-1.30); and Q5, $0.71(0.61-0.83)$. The $P$ values in this model were $<0.001$ (Figure 1B and Supplementary Table 2). The protective effect remained significant after adjusting for age, sex, and other potential confounders; the $P$ values for trends in models 2 and 3 were $<0.001$ and $<0.05$, respectively. Additionally, the abundance of Akkermansia was inversely correlated with the number of MetS components (Figure 1C and D).

Disparities in the effects of Akkermansia were observed among the MetS components. We found that Akkermansia was most closely associated with central obesity and hypertriglyceridemia, followed by reduced HDL-c, and hypertension; notably, no significant associations were found with hyperglycemia in any of the models (Figure 1A and B). For instance, a 1\% increase in Akkermansia abundance significantly reduced the risk of central obesity by $\sim 2 \%$ (model 1 , $\mathrm{OR}=0.983$, 95\% CI: 0.975-0.990; model 2, OR=0.973, 95\% CI: 0.972-0.998; and model 3, OR $=0.982$, 95\% CI: 0.989-0.991), while the protective effect against hyperglycemia was not statistically significant (model $1, \mathrm{OR}=1.000$, 95\% CI: 0.993-1.006; model 2, OR $=0.998,95 \%$ CI: 0.991-1.004; and model 3, OR=0.996, 95\% CI: 0.989-1.003) (Figure 1A). When the abundance of Akkermansia was treated as a categorical variable, we obtained similar results - ie, the protective effect was stronger against central obesity and hypertriglyceridemia than against the other components of MetS. ORs for central obesity and hypertriglyceridemia were lower than those for the other components, and $P$ value trends were $<0.001$ in all models (Figure 1B).

Although the protective association observed across quintiles of Akkermansia showed a strong linear trend, we found that it was limited by lower levels of Akkermansia. For example, the effect on MetS for Q2, Q3, and Q4 was not always statistically significant compared to that for Q5 (Figure 1B), suggesting a doseresponse association.

\section{Dose-Response Effect of Akkermansia on MetS}

To investigate how the risk of MetS changes with Akkermansia abundance, we fitted the dose-response curves of Akkermansia on MetS in the models with cubic splines (Figure 2A) and found that the probability of MetS changed nonlinearly with Akkermansia level ( $P_{\text {nonlinear }}$ $<0.05$ ): the distribution curves were flat before $\sim 0.2 \%$ Akkermansia, declined sharply until $\sim 10 \%$, and then flattened once again. The distribution of MetS probability was 
A

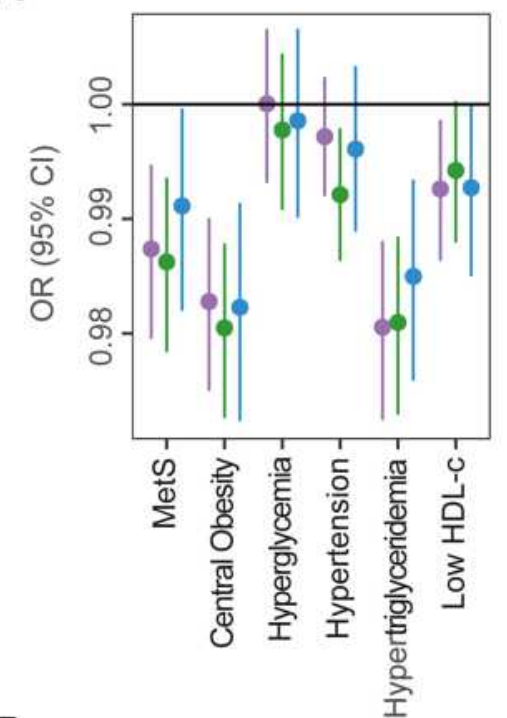

C

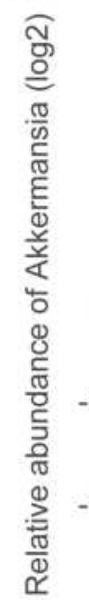

D

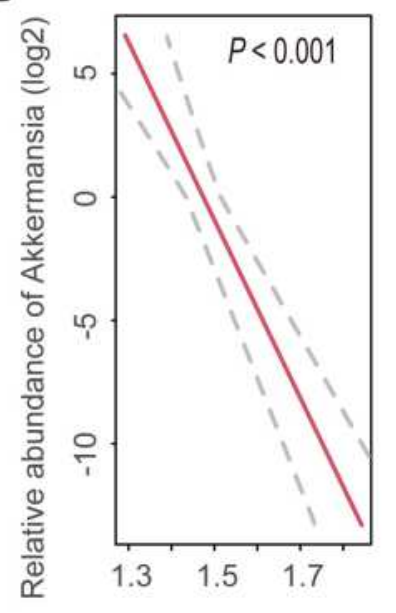

Number of MetS components
Number of MetS components

B
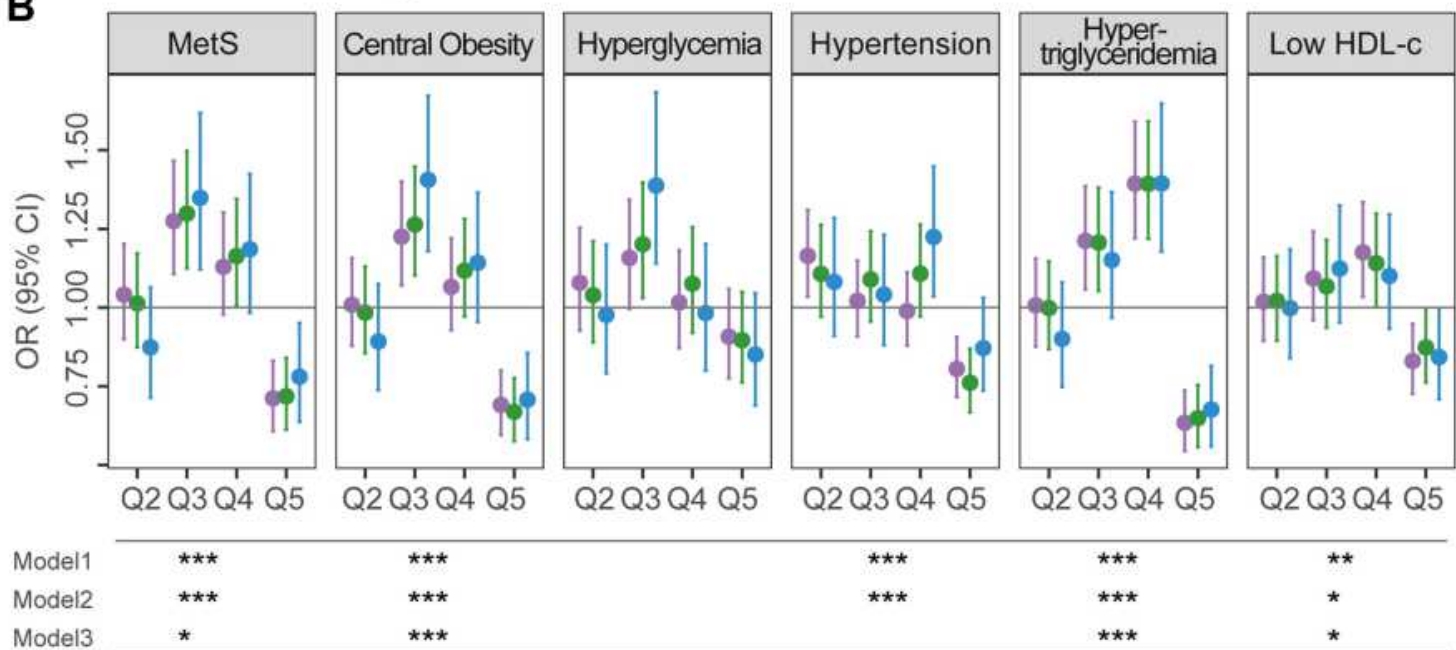

$P$ for trend

* $\leq 0.05$

** $\leq 0.01$

$* * * \leq 0.001$

Figure I Associations between the relative abundance of Akkermansia and MetS and its components. (A and B) ORs and $95 \%$ Cls for MetS and its components with a $1 \%$ increase in the abundance of Akkermansia (A), and across quintiles of Akkermansia (with QI as a reference) (B). (C) Relative abundance of Akkermansia in participants with different numbers of MetS components. (D) Linear association between Akkermansia and the number of MetS components; dashed lines represent the $95 \% \mathrm{Cl}$. Trend test of ORs across quintiles (QI-Q5) was conducted by entering the median value of each quintile of $A k k e r m a n s i a$ abundance into the models. *P for trend $<0.05$; **P for trend $<0.0$ I; ***P for trend $\leq 0.00 \mathrm{I}$.

similar in the 3 models, but varied for different MetS components. To ensure the robustness of this result, we also tested different knots in the models that used splines (Supplementary Figure 3A). Although the inflection point fluctuated according to model parameters, the shape of curves and the nonlinear relationship did not change.

To further evaluate the nonlinear association, we plotted ORs (95\% CIs) of 20 quantiles of Akkermansia in age- and sex-adjusted logistic regression models and examined how the risk of MetS changed with a $1 \%$ increase in Akkermansia in each quantile (Figure 2B and Supplementary Table 3). In accordance with the fitted curves, we found that ORs did not decrease markedly until Akkermansia abundance was $0.1 \%-0.2 \%$, and the protective effect was statistically significant until $\sim 3.8 \%$ $(\mathrm{OR}=0.68,95 \%$ CI: $0.463-1.002)$. The inflection points at which the protective effects of Akkermansia became statistically significant varied among MetS components and models (Supplementary Figure 3B and Supplementary Table 3).

We next assessed the risk of MetS and its components in subjects with high vs low levels of Akkermansia (Table 2; cutoff values were set to 0.1 (Figure 2A) and 0.2 (Figure 2B). The results showed that the risk of MetS and its components 


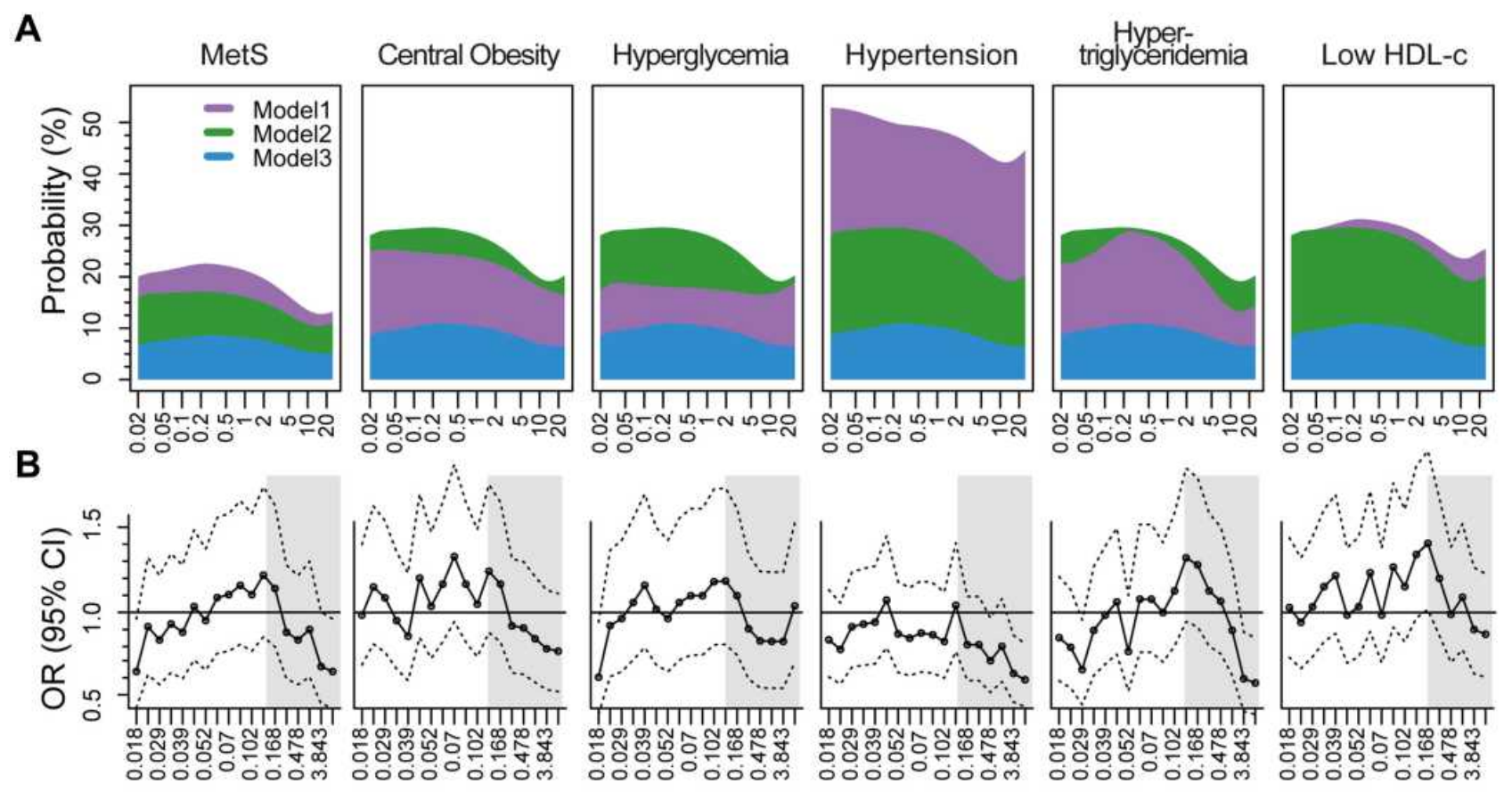

Relative abundance of Akkermansia (\%)

Figure 2 Dose-response effect of Akkermansia on MetS and its components. (A) Association between risk of MetS and relative abundance of Akkermansia. Dose-response curves of Akkermansia were fitted to logistic models with cubic splines. Four knots at the 5th, 25th, 75th, and 95th percentiles were used in models. Model I was a crude regression without adjusting for confounders; model 2 was adjusted for age and sex; and model 3 was further adjusted for Bristol stool scale, geographic location, and annual income. A trend test of ORs across quintiles (QI-Q5) was conducted by entering the median value of each quintile of $A k k e r m a n s i a$ abundance into the models. (B) ORs and 95\% Cls across 20 percentiles of Akkermansia (with the first quintile [QI] as a reference) in crude logistic models.

was reduced to a greater extent when the Akkermansia cutoff was set to $0.2 \%$ as compared to $0.1 \%$.

Taken together, we found that the association between Akkermansia and MetS was nonlinear; dose-response analyses showed that the risk of MetS was not significantly reduced until Akkermansia abundance was $0.2 \%$ of total gut microbiota.

\section{Effects of Microbial Interactions on the Akkermansia-MetS Relationship}

Microbial competition and cooperation affect microbehost interactions in the gut. ${ }^{13,22}$ We therefore investigated whether the Akkermansia-MetS relationship changed with the relative abundance of other bacteria. Three

Table 2 Risks of MetS and Its Components in Subjects with Higher Akkermansia Compared to Those with Lower Akkermansia (or, 95\% Cl) ${ }^{\mathrm{a}}$

\begin{tabular}{|c|c|c|c|c|c|c|}
\hline & MetS & Central Obesity & Hyperglycemia & Hypertension & Hypertriglyceridemia & Low HDL \\
\hline \multicolumn{7}{|l|}{ Cutoff $=0.1^{\mathrm{b}}$} \\
\hline Prevalence (\%) Akk<0.1/Akk $\geq 0.1^{c}$ & $20.9 / 19.6$ & $25.2 / 21.9$ & $18.2 / 17.9$ & $52.2 / 48.4$ & $23.5 / 23.8$ & $28.7 / 28.9$ \\
\hline Model I & $0.99(0.98-1.02)$ & $0.97(0.95-0.99)$ & $0.99(0.98-1.01)$ & $0.96(0.94-0.99)$ & $1.00(0.98-1.02)$ & $1.00(0.98-1.02)$ \\
\hline Model 2 & $0.99(0.97-1.01)$ & $0.97(0.95-0.99)$ & $1.00(0.98-1.02)$ & $0.98(0.99-1.00)$ & $1.00(0.99-1.02)$ & $1.00(0.98-1.03)$ \\
\hline Model 3 & $1.00(0.98-1.02)$ & $0.98(0.95-1.00)$ & $1.00(0.97-1.01)$ & $1.01(0.99-1.04)$ & $1.00(0.98-1.04)$ & $1.00(0.97-1.02)$ \\
\hline \multicolumn{7}{|l|}{ Cutoff $=0.2$} \\
\hline Prevalence (\%) Akk<0.2/Akk $\geq 0.2^{c}$ & $21.7 / 17.3$ & $25.5 / 19.9$ & $18.6 / 17.9$ & $52.1 / 48.9$ & $24.9 / 20.7$ & $29.6 / 26.9$ \\
\hline Model I & $0.96(0.94-0.98)$ & $0.94(0.92-0.96)$ & $0.98(0.96-1.00)$ & $0.95(0.92-0.97)$ & $0.96(0.93-0.98)$ & $0.97(0.95-1.00)$ \\
\hline Model 2 & $0.96(0.94-0.98)$ & $0.94(0.93-0.97)$ & $0.99(0.97-1.00)$ & $0.96(0.94-0.98)$ & $0.96(0.94-0.98)$ & $0.98(0.95-1.00)$ \\
\hline Model 3 & $0.98(0.95-1.00)$ & $0.96(0.93-0.99)$ & $0.97(0.95-1.00)$ & $0.98(0.95-1.00)$ & $0.97(0.94-0.99)$ & $0.98(0.94-1.00)$ \\
\hline
\end{tabular}

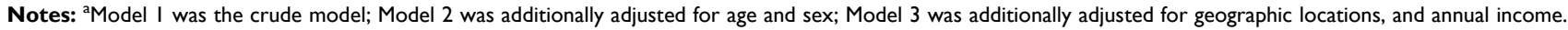

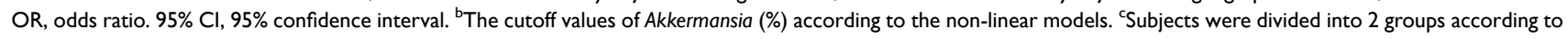
the cutoff abundance of Akkermansia. 
Akkermansia OTUs were annotated according to the Silva_16s_v123 database; all were distributed in $>10 \%$ of samples and were named OTU49, OTU115, and OTU236.

The Akkermansia OTUs had similar distribution patterns (ie, bimodal). However, their associations with MetS differed. OTU49 and OTU115, the 2 major OTUs (Figure 3A), coexisted in $93.7 \%$ of subjects (Figure $3 \mathrm{~B}$ ) but were negatively correlated with each other (Pearson correlation, $\mathrm{r}=-0.037$, $\mathrm{FDR}=0.02$ ) (Figure 3C).

Associations of gut microbes with Akkermansia OTUs are shown as Figure 3D and Supplementary Table 4. We detected 717 significant associations (406 positive $[+]$ and 311 negative [-]) with Akkermansia OTUs after controlling the FDR $(<0.05)$. These included 548 unique OTUs, 161 genera, and 63 bacterial families. At the family level, Ruminococcaceae had the most associations $(n=167)$, followed by Lachnospiraceae $(n=139)$, Bacteroidaceae $(n=35)$, and Prevotellaceae $(n=35)$. Interestingly, we found that the type of association ( + or - ) was largely consistent within the same family. For instance, Akkermansia was positively associated with most
Ruminococcaceae (127+ and 40-) and Bacteroidaceae (23+ and 12-) but negatively associated with most Lachnospiraceae (36+ and 103-) and Prevotellaceae (4+ and 22-). At the genus level, Bacteroides, Lachnoclostridium, and Alistipes were the 3 most abundant bacteria, with 35 (23+ and 12-), 24 (7+ and 17-), and 21 (21+ and $0-)$ associations, respectively.

Of the 3 Akkermansia OTUs, only OTU49 was significantly associated with MetS (Figure 4A). We further examined the microbes that potentially influence the association between the OTU49 and MetS. The analysis of interactions showed that 25 iOTUs significantly affected the OTU49-MetS association ( $P$ for interactions $<0.05$ ) and 17 were also significantly associated with OTU49 $(P$ for association <0.05) (Supplementary Table 5). Moreover, most of the iOTUs showed a positive association with OTU49 ( $\beta$ for associations $>0$ ). The iOTUs could be separated into 2 groups according to their effects on the Akkermansia-MetS association ( $\triangle \mathrm{OR}$ ) (Figure 4B). The high abundance of iOTUs in group 1 weakened the protective effect of OTU49 on MetS, while iOTUs from group 2 strengthened the effect. For example,
A

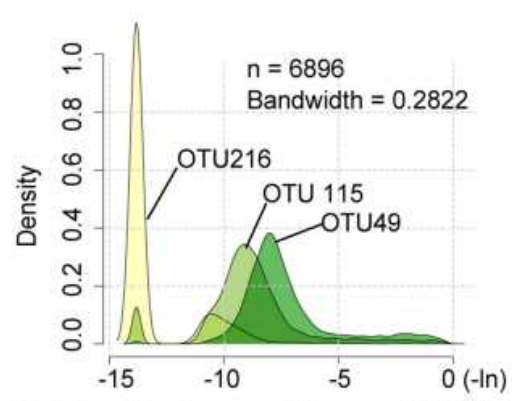

Rellative abundance of Akkermansia OTUs

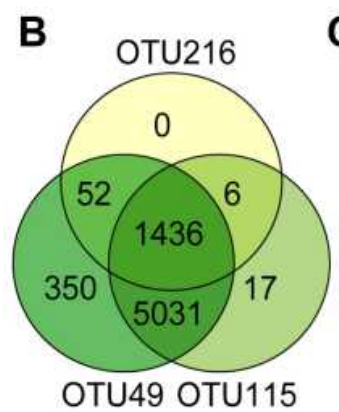

C

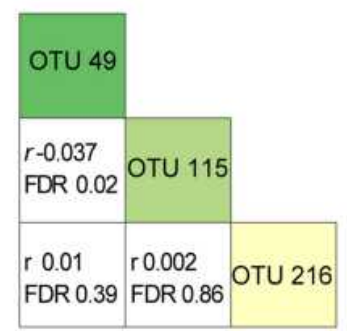

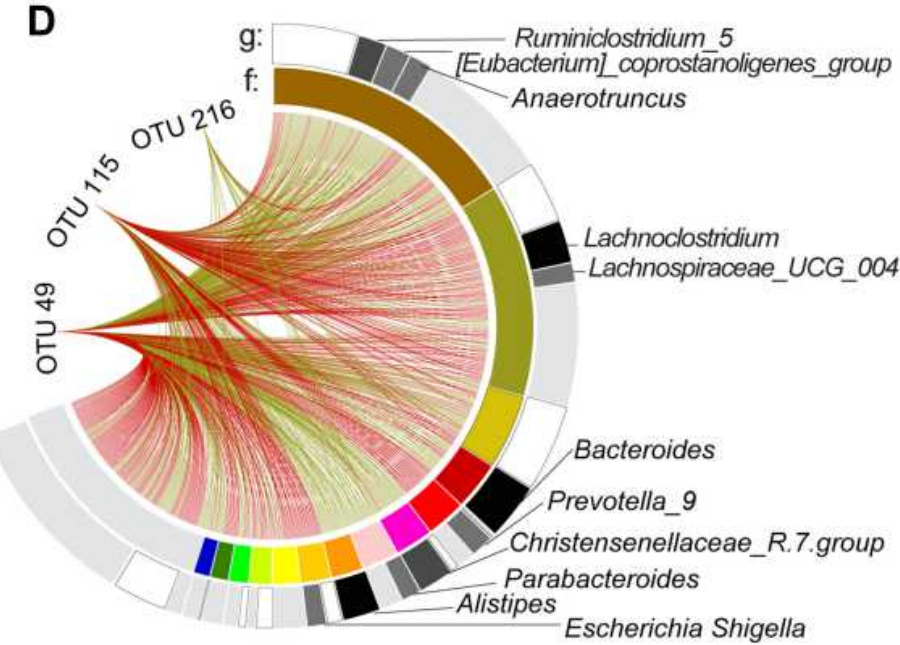

Ruminococcaceae Lachnospiraceae

Unclassified

Bacteroidaceae

Prevotellaceae

Christensenellaceae

Porphyromonadaceae

Rikenellaceae

Figure 3 Distribution of Akkermansia OTUs and their correlations with other microbiota. (A) Distribution of 3 Akkermansia OTUs. (B) Overlap of the distributions of Akkermansia OTUs in all participants. (C) Correlations of the 3 Akkermansia OTUs. $r$ is the Pearson coefficient. (D) Associations between Akkermansia OTUs and other gut microbes. The first and second circles represent OTUs at the genus (g) and family (f) levels, respectively; and red and green lines represent positive and negative correlations, respectively. Only significant correlations are shown. 
A

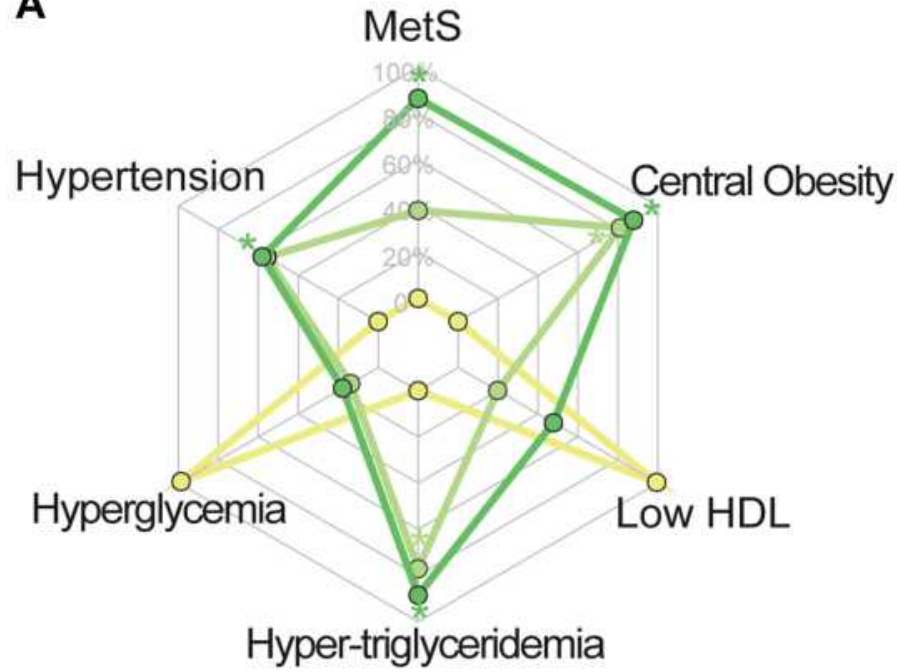

C

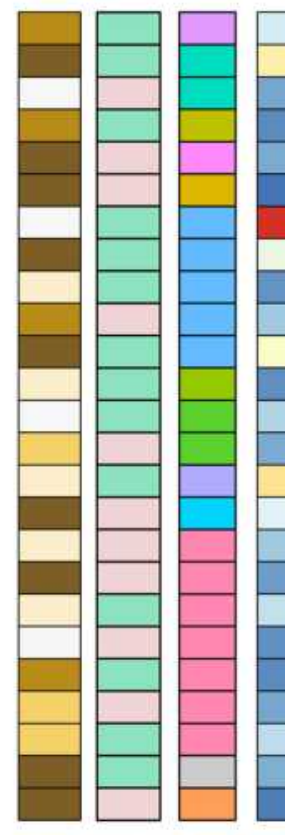
OTU5906 g:Escherichia-Shigella
OTU125 g:Parasutterella OTU2425 g:Bacteroides OTU2824 g:Bacteroides OTU678 g:Rheinheimera OTU5225 g:Family XIII UCG-001 OTU166 g:Blautia OTU140 g:Dorea OTU1924 g:Lachnoclostridium OTU160 g:Lachnospiraceae UCG-001 OTU266 g:Unclassified OTU801 g:Micrococcus OTU10015 g:Unclassified OTU10026 g:Unclassified OTU885 g:Acinetobacter OTU485 g:Barnesiella OTU182 g:[Eubacterium] coprostanoligenes group OTU8016 g:Anaerotruncus OTU1460 g:Faecalibacterium OTU548 g:Ruminiclostridium 9 OTU622 g:Ruminococcaceae UCG-005 OTU555 g:Unclassified OTU682 g:Unclassified OTU2912 g:Allisonella OTU1026 g:Unclassified
B



Relative abundance of iOTUs



$\triangle$ OR
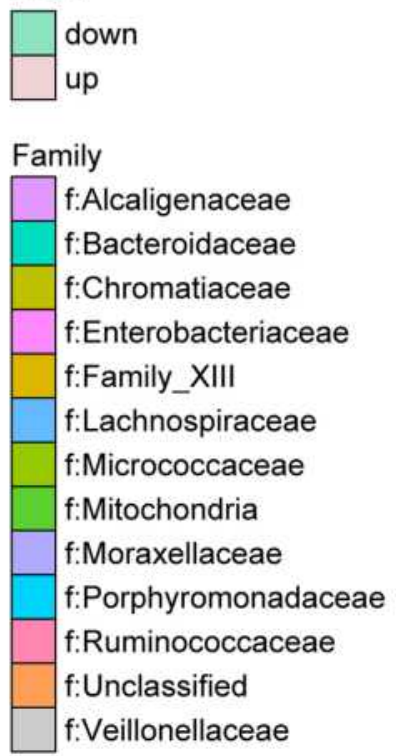

$p \quad \Delta \mathrm{OR}$ Family $\beta$

Figure 4 Effects of microbial interactions on the Akkermansia-MetS association. (A) Associations of MetS and its components with 3 Akkermansia OTUs. (B) Groups of iOTUs according to their effects on the Akkermansia-MetS association. Interactions were visualized by changes in the protective effect of Akkermansia against MetS (ORs) when the abundance of iOTUs increased from QI to Q5. ORs were estimated by logistic regression in which the abundance of $A k k e r m a n s i a$ was treated as a continuous variable. (C) Annotation of iOTUs at the family and genus levels. $\beta$ and FDR were estimated with the multivariate linear regression. Equation: $\Delta O R=O R_{(\text {iOTUat } Q 5)}-O R_{(\text {OOTUatQ } 1)}$.

Akkermansia had a stronger protective effect against MetS when OTU485 was more abundant (Q5, OR=0.083, 95\% CI: $0.011-0.45$ and Q1, OR=0.669, 95\% CI: 0.155-2.406). This strengthened effect persisted when OTU485 was treated as a continuous variable, and the increment of each SD of OTU485 reduced the ORs for MetS (Supplementary Figure 4). The iOTUs were annotated to 18 genera and 12 bacterial families. In line with the above results, Ruminococcaceae and Lachnospiraceae were the 2 predominant families enriched in 7 and 5 genera, respectively (Figure 4C). Four genera in Lachnospiraceae (including Blautia, Dorea, and Lachnoclostridium) and 2 in Ruminococcaceae (including Faecalibacterium and Ruminococcaceae UCG-005) reduced the ORs of Akkermansia for MetS, reflecting a greater protective effect of Akkermansia. On the contrary, Anaerotruncus, 
Lachnospiraceae UCG-001, [Eubacterium] coprostanoligenes group, and Ruminiclostridium-9 potentially diminished the protective effect.

\section{Discussion}

The present study investigated the associations between gut Akkermansia and MetS in a large cohort with adjustment for potential confounders. We found that Akkermansia abundance was differentially associated with MetS components. We also demonstrated for the first time a nonlinear relationship between Akkermansia and MetS and the influence of microbial interactions on this association.

Some studies have reported an association between increased A. muciniphila and reduced prevalence of obesity, untreated T2DM, and hypertension. ${ }^{23-25}$ In this study, we found a significant association between Akkermansia and central obesity/waist circumference, hypertriglyceridemia/TG, reduced HDL-c (as a categorical variable)/HDL-c (as a continuous variable), SPB, and DBP, although it was unrelated to hyperglycemia, FBG, and hypertension. The discrepancy between our results and previous findingsespecially pertaining to FBG and hyperglycemia-may come from the high degree of heterogeneity of the host and variability of gut microbiota. Our analyses were based on a large population and were adjusted for some confounders such as sex, age, and geography, which could potentially reduce the bias caused by confounders.

Our finding of an Akkermansia-obesity association is consistent with another study based on the American Gut Project, which included around 10,000 subjects. ${ }^{26}$ Both studies adjusted for potential confounders such as age, sex, and geographic region, and we observed a significant relationship between Akkermansia and both central and overall obesity. The concordance of these results indicates that the protective effect of Akkermansia against obesity is independent of geography, ethnicity, sex, age, and dietary habits in Western and Eastern countries and may be generalizable to a large population.

Previous studies have reported conflicting results on the associations between Akkermansia and components of MetS. A. muciniphila was shown to control fat storage, adipose tissue metabolism, and glucose homeostasis to reverse diet-induced metabolic disorders in mice and humans. $^{27,28}$ However, another study from the same researchers did not find significant changes in fasting glycemia and HbA1c in human subjects after 3 months of $A$. muciniphila administration, with similar results for insulin sensitivity. ${ }^{12}$ The interaction between lipid or glucose metabolism and Akkermansia has been widely studied, but the differential effects of Akkermansia on the 2 processes has rarely been addressed. ${ }^{29}$ Our epidemiologic results showed that hyperglycemia had a weaker association with Akkermansia than obesity and hypertriglyceridemia, suggesting differences between lipid-Akkermansia and glucose-Akkermansia interactions. The protective mechanisms of Akkermansia against metabolic disorders include increasing mRNA expression of markers of adipocyte differentiation and lipid oxidation; counteracting colon mucus suppression induced by a high-fat diet; and reducing hepatic glucose-6-phosphatase and increasing acylglycerols in the ileum. ${ }^{7,27}$ However, the interaction of lipid and glucose metabolism under the regulation of gut bacteria requires more detailed investigation. Our results also suggest the need for preventive strategies targeting different components of MetS.

While most gut microbes showed significant correlations with Akkermansia, only a few OTUs influenced the Akkermansia-MetS association. A previous study found that the gut microbiome of mice was unaltered after Akkermansia gavage for 4 weeks. ${ }^{27}$ On the other hand, 2 studies reported an increase in Bifidobacterium abundance following Akkermansia administration, ${ }^{30,31}$ which is inconsistent with our results. We found that Ruminococcaceae and Lachnospiraceae were the 2 main families influencing Akkermansia abundance and the Akkermansia-MetS association. Microbial interactions with Akkermansia and their modulation of MetS have rarely been studied, and interactions between different species can vary across environmental conditions, space, and potentially evolutionary time. ${ }^{13}$ Thus, additional studies are needed to clarify the relationships between Akkermansia, other gut microbes, and their hosts.

Our study identified the effective dose of Akkermansia for protection against MetS. The protective effect of Akkermansia was nonlinear and nonsignificant at a low relative abundance, which is in line with previous reports; a dose of $10^{8} \mathrm{CFU} /$ day was found to be required to prevent or treat metabolic disorders, ${ }^{32}$ whereas one study showed that a daily $A$. muciniphila dose $>4 \times 10^{7} \mathrm{CFU}$ was necessary in mice. ${ }^{33}$ Although the exact threshold level of Akkermansia could not be determined due to the limitations of $16 \mathrm{~S}$ rRNA sequencing, our results suggest that the risk of MetS is only reduced when Akkermansia represents at least of $0.2 \%$ of the whole gut microbiota. 
We also provide epidemiologic evidence for the safety of Akkermansia in the Chinese population. Akkermansia is widely distributed in the human gut; $>95 \%$ of the population harbors Akkermansia and $\sim 20 \%$ has an abundance $>7.05 \%$. A similar distribution has been reported in the American and European populations: Akkermansia was detected in $75 \%$ of European samples, ${ }^{34} 82 \%$ of British samples, ${ }^{26}$ and $83 \%$ of American samples; ${ }^{26}$ and the median abundance of Akkermansia was $0.08 \%$ (IQR: $0.04-0.34 \%$ ) and $0.08 \%$ (IQR: $0.006-0.93 \%$ ) in Chinese and the American samples, ${ }^{27}$ respectively. The protective effects of Akkermansia increased with its abundance and no adverse effects were observed when it was present at high levels. There have been few clinical studies demonstrating the safety of Akkermansia, but an interventional study reported a tolerable A. muciniphila dose of $10^{10} \mathrm{CFU} /$ day in overweight or obese individuals, ${ }^{12,35}$ while another showed no adverse effects even when the proportion of A. muciniphila was $60 \%$ of total human gut bacteria. ${ }^{36}$

It is worth noting that we performed analyses at the OTU and genus levels of Akkermansia because of the limitations of $16 \mathrm{~S}$ rRNA sequencing. In fact, there are at least 8 different species reported in this genus, ${ }^{37}$ although only one A. muciniphila strain (MucT) is available and widely used for intervention in metabolic disorders. The associations between MetS and Akkermansia at genus and species or strain levels have been previously reviewed; ${ }^{33}$ however, it remains unclear how these differ across species. In the present study, we detected 3 Akkermansia-like OTUs but only one showed significant associations with MetS and its components.

A strength of our study is that it provides evidence for the relationship between Akkermansia and MetS; the large sample size allowed us to estimate the independent protective effect of Akkermansia by adjusting for several potential confounders. Moreover, this is the first demonstration of an effective dose of Akkermansia for protection against MetS and the first report to highlight the contribution of other gut microbes on this effect.

Our study had certain limitations. Firstly, the results were based on a Chinese population and may not be completely generalizable to Western subjects; that is, the effective dose of Akkermansia may differ given the disparities in MetS prevalence and Akkermansia abundance across different geographic regions or ethnic groups. Secondly, there may be methodologic bias associated with 16S rRNA sequencing, which is commonly used to detect bacteria at the genus level; shotgun metagenomic sequencing or pure culture-based analyses at the species or strain level should therefore be performed. Finally, the lack of some medication records may have biased the result. For example, missing 2-h postprandial blood glucose data may have affected the definition of hyperglycemia; and metformin intake can increase Akkermansia abundance $^{38}$ but was not included in this dataset. Accordingly, the present study provides only cross-sectional evidence for the Akkermansia-MetS relationship.

\section{Conclusion}

The results of this study suggest that a higher abundance of Akkermansia was associated with a lower risk of MetS in the Chinese population, whereas the associations with different MetS components varied. We also found that the protective effect of Akkermansia is influenced by microbial interactions and may not be significant below a certain abundance threshold. These findings suggest that probiotic intervention with Akkermansia can prevent or alleviate MetS and related disorders such as heart disease or T2DM.

\section{Acknowledgments}

This study was supported by the National Scientific Foundation of P. R. China [91849132, 81571385, 81870552 and 81400790], Non-profit Central Research Institute Fund of Chinese Academy of Medical Sciences [2018RC330003], CAMS Innovation Fund for Medical Sciences [2018-I2M1 -002], the Beijing Hospital Nova Project [BJ-2018-139], National Key R\&D Program of China [2018YFC2000400], Priority Union Foundation of Yunnan Provincial Science and Technology Department and Kunming Medical University [202001AY070001-011], and Guangxi fund project on natural science [2018GXNSFAA138156]. The funders had no role in study design, data collection and analysis, decision to publish, or preparation of the manuscript.

We thank Dr. Yong Jia from Murdoch University, Xiaoquan Zhu from Beijing Hospital, and Dr. Jun Xiong from Institute of Biophysics, Chinese Academy of Sciences for their continuous support during the preparation of the manuscript.

\section{Disclosure}

The authors report no conflicts of interest in this work.

\section{References}

1. Saklayen MG. The global epidemic of the metabolic syndrome. Curr Hypertens Rep. 2018;20(2):12. doi:10.1007/s11906-018-0812-z 
2. Dabke K, Hendrick G, Devkota S. The gut microbiome and metabolic syndrome. J Clin Invest. 2019;129(10):4050-4057. doi:10.1172/JCI129194

3. Gildner TE. Links between metabolic syndrome and the microbiome. Evol Med Public Health. 2020;2020(1):45-46. doi:10.1093/emph/ eoaa007

4. Le Chatelier E, Nielsen T, Qin J, et al. Richness of human gut microbiome correlates with metabolic markers. Nature. 2013;500 (7464):541-546. doi:10.1038/nature12506

5. Chen X, Devaraj S. Gut microbiome in obesity, metabolic syndrome, and diabetes. Curr Diabetes Rep. 2018;18(12):129. doi:10.1007/ s11892-018-1104-3

6. Roy CC, Kien CL, Bouthillier L, Levy E. Short-chain fatty acids: ready for prime time? Nutr Clin Pract. 2006;21(4):351-366. doi:10.1177/0115426506021004351

7. Fan Y, Pedersen O. Gut microbiota in human metabolic health and disease. Nat Rev Microbiol. 2021;19(1):55-71. doi:10.1038/s41579020-0433-9

8. Zhang T, Li Q, Cheng L, et al. Akkermansia muciniphila is a promising probiotic. Microb Biotechnol. 2019;12(6):1109-1125. doi:10.1111/1751-7915.13410

9. Cani PD, de Vos WM. Next-generation beneficial microbes: the case of Akkermansia muciniphila. Front Microbiol. 2017;8:1765. doi:10.3389/fmicb.2017.01765

10. Naito Y, Uchiyama K, Takagi T. A next-generation beneficial microbe: Akkermansia muciniphila. J Clin Biochem Nutr. 2018;63 (1):33-35. doi:10.3164/jcbn.18-57

11. Anhê FF, Pilon G, Roy D, Desjardins Y, Levy E, Marette A. Triggering Akkermansia with dietary polyphenols: a new weapon to combat the metabolic syndrome? Gut Microbes. 2016;7(2):146-153. doi:10.1080/19490976.2016.1142036

12. Depommier C, Everard A, Druart C, et al. Supplementation with Akkermansia muciniphila in overweight and obese human volunteers: a proof-of-concept exploratory study. Nat Med. 2019;25 (7):1096-1103. doi:10.1038/s41591-019-0495-2

13. Coyte KZ, Rakoff-Nahoum S. Understanding competition and cooperation within the mammalian gut microbiome. Curr Biol. 2019;29 (11):R538-R544. doi:10.1016/j.cub.2019.04.017

14. Geerlings S, Kostopoulos I, De Vos W, et al. Akkermansia muciniphila in the Human gastrointestinal tract: when, where, and how? Microorganisms. 2018;6(3):75. doi:10.3390/microorganisms6030075

15. He Y, Wu W, Wu S, et al. Linking gut microbiota, metabolic syndrome and economic status based on a population-level analysis. Microbiome. 2018;6(1):172. doi:10.1186/s40168-018-0557-6

16. He Y, Wu W, Zheng HM, et al. Regional variation limits applications of healthy gut microbiome reference ranges and disease models. Nat. Med. 2018;24(10):1532-1535. doi:10.1038/s41591-018-0164-x

17. Joint committee for guideline revision. 2016 Chinese guidelines for the management of dyslipidemia in adults. $J$ Geriatr Cardiol. 2018;15(1):1-29. doi:10.11909/j.issn.1671-5411.2018.01.011.

18. Magoč T, Salzberg SL. FLASH: fast length adjustment of short reads to improve genome assemblies. Bioinformatics. 2011;27 (21):2957-2963. doi:10.1093/bioinformatics/btr507

19. Rognes T, Flouri T, Nichols B, Quince C, Mahé F. VSEARCH: a versatile open source tool for metagenomics. PeerJ. 2016;4: e2584. doi:10.7717/peerj.2584

20. Quast C, Pruesse E, Yilmaz P, et al. The SILVA ribosomal RNA gene database project: improved data processing and web-based tools. Nucleic Acids Res. 2013;41(D1):D590-596. doi:10.1093/nar/gks1219

21. Gauthier J, Wu QV, Gooley TA. Cubic splines to model relationships between continuous variables and outcomes: a guide for clinicians. Bone Marrow Transplant. 2020;55(4):675-680. doi:10.1038/s41409019-0679-x
22. Knights D, Ward TL, McKinlay CE, Miller H, Gonzalez A. Rethinking "Enterotypes". Cell Host Microbe. 2014;16(4):433-437. doi:10.1016/j.chom.2014.09.013

23. Brahe LK, Chatelier EL, Prifti E, et al. Specific gut microbiota features and metabolic markers in postmenopausal women with obesity. Nutr Diabetes. 2015;5(6):e159. doi:10.1038/nutd.2015.9

24. Yassour M, Lim MY, Hyun S, et al. Sub-clinical detection of gut microbial biomarkers of obesity and type 2 diabetes. Genome Med. 2016;17(8):8. doi:10.1186/s13073-016-0271-6

25. Li J, Zhao F, Wang Y, Chen J, Tao J, Tian G. Gut microbiota dysbiosis contributes to the development of hypertension. Microbiome. 2017;5(1):14. doi:10.1186/s40168-016-0222-x

26. Zhou Q, Zhang Y, Wang X, et al. Gut bacteria Akkermansia is associated with reduced risk of obesity: evidence from the American Gut Project. Nut Metab (Lond). 2020;17(90). doi:10.1186/s12986-020-00516-1

27. Amandine E, Clara B, Lucie G, et al. Cross-talk between Akkermansia muciniphila and intestinal epithelium controls diet-induced obesity. Proc Natl Acad Sci USA. 2013;110 (22):9066-9071. doi:10.1073/pnas.1219451110

28. Dao MC, Everard A, Aronwisnewsky J, et al. Akkermansia muciniphila and improved metabolic health during a dietary intervention in obesity: relationship with gut microbiome richness and ecology. Gut. 2016;65(3):426-436. doi:10.1136/gutjnl-2014-308778

29. Xu Y, Wang N, Tan HY, Li S, Zhang C, Feng Y. Function of Akkermansia muciniphila in obesity: interactions with lipid metabolism, immune response and gut systems. Front Microbiol. 2020;11:219. doi:10.3389/fmicb.2020.00219

30. Org Parks BW, Joo JW, Emert B, et al. Genetic and environmental control of host-gut microbiota interactions. Genome Res. 2015;25 (10):1558-1569. doi:10.1101/gr.194118.115

31. Chevalier C, Stojanović O, Colin D, et al. Gut microbiota orchestrates energy homeostasis during cold. Cell. 2015;163(6):1360-1374. doi:10.1016/j.cell.2015.11.004

32. Derrien M, Belzer C, de Vos WM. Akkermansia muciniphila and its role in regulating host functions. Microb Pathog. 2017;106:171-181. doi:10.1016/j.micpath.2016.02.005

33. Shin NR, Lee JC, Lee HY, et al. An increase in the Akkermansia spp. population induced by metformin treatment improves glucose homeostasis in diet-induced obese mice. Gut. 2014;63(5):727-735 doi:10.1136/gutjnl-2012-303839

34. Collado MC, Derrien M, Isolauri E, et al. Intestinal integrity and Akkermansia muciniphila, a mucin-degrading member of the intestinal microbiota present in infants, adults, and the elderly. Appl Environ Microbiol. 2007;73(23):7767. doi:10.1128/AEM.01477-07

35. Plovier H, Everard A, Druart C, et al. A purified membrane protein from Akkermansia muciniphila or the pasteurized bacterium improves metabolism in obese and diabetic mice. Nat Med. 2017;23 (1):107-113. doi: $10.1038 / \mathrm{nm} .4236$

36. Dubourg G, Lagier JC, Armougom F, et al. High-level colonisation of the human gut by Verrucomicrobia following broad-spectrum antibiotic treatment. Int $J$ Antimicrob Agents. 2013;41(2):149-155. doi:10.1016/j.ijantimicag.2012.10.012

37. Passel MWJ, Van, Kant R, Zoetendal EG, et al. The genome of Akkermansia muciniphila, a dedicated intestinal mucin degrader, and its use in exploring intestinal metagenomes. PLoS One. 2011;6 (3):e16876. doi:10.1371/journal.pone.0016876

38. Qin J, Li Y, Cai Z, et al. A metagenome-wide association study of gut microbiota in type 2 diabetes. Nature. 2012;490(7418):55-60. doi:10.1038/nature11450 


\section{Publish your work in this journal}

Diabetes, Metabolic Syndrome and Obesity: Targets and Therapy is an international, peer-reviewed open-access journal committed to the rapid publication of the latest laboratory and clinical findings in the fields of diabetes, metabolic syndrome and obesity research. Original research, review, case reports, hypothesis formation, expert opinion and commentaries are all considered for publication. The manuscript management system is completely online and includes a very quick and fair peer-review system, which is all easy to use. Visit http://www.dovepress.com/testimonials.php to read real quotes from published authors.

Submit your manuscript here: https://www.dovepress.com/diabetes-metabolic-syndrome-and-obesity-targets-and-therapy-journal 